\title{
L'AFFIXE, LA RACINE, ET LA LINGUISTIQUE ANALYTIQUE
}

\author{
François Jacquesson \\ CNRS Lacito (UMR7107) et Sorbonne Nouvelle, Paris, France
}

\section{Résumé}

Les termes de 'racine', 'affixe', puis 'suffixe' et 'préfixe' n'appartiennent pas au matériel lexical créé pendant l'Antiquité, mais comme celui de 'scheva' ou 'shewa', résultent de la découverte de la grammaire hébraïque par les grammairiens de langue latine, à la Renaissance. Ces mots nouveaux, dont on examine ici la naissance et le développement, nous semblent caractéristiques d'un nouveau type d'approche des langues. Pour la première fois, les grammairiens de langue latine sont confrontés à une tradition étrangère, vivante et prestigieuse. La tradition grammaticale hébraïque, qu'ils découvrent, leur fournit aussi un champ de réflexion et des outils d'analyse. De cette rencontre avec une langue et avec sa tradition critique, qui offre vaste matière à comparaisons, vont sortir des termes nouveaux et une perspective renouvelée sur l'analyse morphologique des langues.

\section{Mots-clés}

linguistique descriptive, morphologie, histoire des concepts linguistiques, linguistique analytique, racine, affixe, histoire de la grammaire hébraïque, Renaissance

\begin{abstract}
'Root' and 'affix' as linguistic terms, and later 'suffix' and 'prefix', do not belong to the large lexical technical lore inherited from the Greco-Latin world. Like 'scheva, shewa', they appear during the Renaissance, when grammarians from the Latin tradition discover Hebrew grammar. For the first time, 'Latin grammarians' have to tackle with a foreign but prestigious tradition. The history of these new words is here described with some detail, because they also reflect a new look at languages. The confrontation between the two grammatical traditions will result in the development of new concepts and a new, more analytical, approach to language morphology.
\end{abstract}

\section{Keywords}

descriptive linguistics, morphology, history of linguistics notions, analytic linguistics, root, affix, history of Hebrew grammar, Renaissance

\section{INTRODUCTION}

Une grande part de notre terminologie grammaticale provient des grammairiens anciens, grecs et latins. Cet héritage doit être visité avec précaution : la pérennité du lexique n'implique pas celle de l'emploi qu'on en fait, et d'autres mots importants, porteurs de conceptions différentes, sont venus modifier la pratique grammaticale, et même en modifier l'ambition: la recherche en linguistique, ce n'est plus la grammaire.

Un pivot dans cette histoire, nous semble-t-il, est la Renaissance, quand cette part de la réflexion sur le langage qui est impliquée dans la «description» de la 
langue se trouve transformée par le contact avec des langues sans rapport avec le latin ou le grec anciens. Vu de près, on peut avoir l'impression que c'est en gros la même démarche qui continue de s'appliquer, avec les mêmes catégories. Mais si l'on observe sur la longue durée ce qui a pu changer, sous la pérennité d'une part de la terminologie on observe par étapes une attitude différente à l'égard des langues : plus expérimentale (partie 1).

Cette attitude nouvelle se marque en particulier par l'émergence de deux concepts nouveaux, 'racine' et 'affixe', deux concepts complémentaires et tous deux empruntés à la grammaire de l'hébreu ${ }^{1}$ - la première langue «nouvelle» à laquelle les linguistes de la Renaissance aient été puissamment confrontés (partie 2). La maturation de ces deux concepts, leur lente excursion au-delà de cette rencontre latino-hébraïque, est évoquée ensuite ${ }^{2}$ (partie 3).

\section{La Linguistique ANALYTiQue}

Plusieurs ${ }^{3}$ concepts fondamentaux de ce que nous appelons ici la «linguistique analytique $^{4} \gg$ ne sont apparus qu'à la Renaissance: au moment où la grammaire de tradition classique se confronte pour la première fois, dans des conditions particulières, à une langue différente. La terminologie nouvelle de ces concepts est discrète, ne tranche pas sur l'héritage antique, mais son propos est différent. Il ne s'agit plus seulement de décrire des classes de mots ou leurs fonctions dans une langue connue, mais d'essayer de segmenter des mots inconnus pour en comprendre à la fois la formation et le fonctionnement; d'où ce nom proposé de «linguistique analytique», qu'on décrit souvent à tort comme une «linguistique descriptive».

1 Nous avons suivi ici les conventions ordinaires pour translittérer l'hébreu postbiblique, mais n'avons pas noté le redoublement de la consonne après l'article quand celui-ci est séparé par un trait d'union; les traits sur ou sous $\mathrm{p}, \mathrm{t}, \mathrm{k}$ notent des prononciations fricatives, non pas des graphies distinctes. Les grammairiens de langue latine tantôt écrivent avec l'alphabet hébreu (auquel cas nous translittérons en italiques), tantôt transcrivent ou translittèrent en alphabet latin (auquel cas nous laissons dans leur texte latin l'hébreu en romain). Tous les textes latins sont traduits par nous.

2 Cet article n'est pas la présentation de découvertes originales concernant l'histoire de la grammaire hébraïque ou latine. La découverte, par les savants de la langue latine, de la tradition grammaire hébraïque, a fait l'objet de plusieurs travaux, notamment ceux de Sophie KesslerMesguish, qui a consacré plusieurs pages importantes (2003, p. 204-206) aux notions d'affixe et de racine. Cet article est un effort pour mettre ces travaux particuliers dans une perspective plus générale, et tenter de voir l'histoire des connaissances sur le long terme.

3 L'auteur remercie Bernard Colombat et Aimée Lahaussois qui ont lu un travail préliminaire, les collaborateurs de la revue HEL qui ont aidé à donner une forme meilleure à cet article, en particulier Judith Kogel pour ses conseils à propos de l'hébreu, et Valérie Raby pour ses encouragements.

4 Cette dénomination n'a pas d'autre prétention que de souligner la continuité d'une attitude expérimentale, et donc d'une perspective théorique, pour une part des sciences du langage. Les raisons du choix de «analytique» apparaîtront plus clairement au cours de l'article; nous ne souhaitons suggérer aucun rapport spécial avec les nombreuses autres disciplines qui ont été, pour des raisons diverses, dites « analytiques». 
$\mathrm{Au}$ premier rang des outils nouveaux se trouvent les notions de «racine» et d' " affixe », utiles aux opérations de segmentation qui vont transformer l'approche des langues, et créditer à terme la grammaire comparée, puis les pratiques actuelles. L'idée de la grammaire comparée repose sur la possibilité d'identifier les éléments comparables, par le rapprochement de formes segmentables dans des langues distinctes et parfois très différentes les unes des autres dans leur aspect moderne. Il s'agit donc de pratiquer des opérations sur les éléments virtuellement indépendants, pour se mettre en mesure d'en examiner séparément la trajectoire historique. Cette 'dissection opératoire' n'est possible que par l'invention combinée des notions de «racine» et d' «affixe» qui visent à observer d'abord le fonctionnement morphologique.

La linguistique « de découverte » qui nous permet d'aborder de façon intelligible des langues de plus en plus nombreuses repose également sur des opérations de substitution ou de réduction qui visent notamment à identifier des combinatoires possibles en morphologie comme en syntaxe, et aussi à distinguer ce qui est théoriquement possible de ce qui est pratiqué en fait. L'outil générique majeur est celui de la segmentation, afin de déterminer des unités de composition à différents niveaux, et leur jeu ensemble. C'est pourquoi les concepts de «racine» et d'«affixe» se révèlent si décisifs, dans la mesure surtout où ils ont pu être généralisés à une gamme croissante de situations.

Cette approche morphologique va, à certains égards, se substituer à l'approche par les catégories lexicales («parties du discours»), notamment à cause du problème récurrent que pose l'opposition discutée entre nom et verbe, ou le caractère parfois affixable, y compris en «personnes », de catégories jusque-là tenues pour invariables, par exemple les prépositions. En pratique, l'approche morpho-syntaxique allait permettre d'analyser des langues inconnues jusqu'alors. C'est une pratique analogue à celle de la dissection. Elle ne signifie pas qu'on ignore les fonctions des organes, mais elle signale qu'on ne peut pas les présumer.

C'est cette approche analytique qui, en mettant en corrélation la variété des langues et le problème de «l'enquête » à leur égard, allait plus tard rendre possible conjointement la prise en compte de la diversité des langues et de la variété des formes, par exemple à travers des catalogues et atlas, et à travers cela l'idée d'une linguistique générale.

\section{UN VOCABULAIRE EMPRUNTÉ}

\subsection{La racine}

Ce mot de 'racine' est nouveau. Tissard en 1509 avait été le premier à l'employer en latin ${ }^{5}$. Reuchlin avait utilisé le Sep̄er ha-šorašim 'le Livre des racines' de David

5 Voir l'étude du travail de Tissard dans Kessler-Mesguich 2013, p. 99-120; emploi de radix p. 119. 
Qimhi, puisque c'est alors l'ouvrage de référence en lexicographie hébraïque. C'est donc vers cet ouvrage que leurs collègues, Farissol, Loans, Sforno puis Elie Levita, avaient guidé les grammairiens de langue latine, Tissard, Reuchlin, Pellikan, Münster, et d'autres encore. Mais Münster, dans la préface de son Dictionarium hebraicum de 1523, précise à plusieurs reprises : liber de Radicibus, sic enim illa gens themata dictionum vocat 'le Livre sur les racines, car c'est ainsi que ces gens appellent les thèmes des mots'. Le mot de 'racine' est, pour les grammairiens de langue latine, un mot nouveau dans ce sens.

Buxtorf un siècle plus tard, dans son Epitome grammaticae hebraeae pour les écoles, dont toutes les éditions portent l'Avant-propos de 1605, utilise couramment le mot thema pour désigner le radical tri-consonantique du verbe hébreu; par exemple dans un titre: Regulae de themate facile investigando et cognoscendo 'Règles pour reconnaitre et identifier facilement le radical'. Au chap. 6 de ce manuel, il explique que les Grecs appellent cela $\theta \dot{\varepsilon} \mu \alpha$ [théma] 'thème' et les Hébreux שירוֹ [šoreš] 'racine'. Mais au temps de Buxtorf, la notion grammaticale de 'racine' est devenue courante chez les savants.

Le mot grec théma, dans son sens grammatical, était attesté chez le grammairien grec antique Apollonios Dyscole ${ }^{6}$ (dont le livre majeur est publié à Venise en 1495), où il apparaît surtout quand la régularité de la flexion (akolouthia) est discutable, notamment chez les pronoms, et qu'il faut accepter (ou non) une diversité de 'thèmes', ce que Lallot compare au supplétisme.

En revanche, le mot 'racine' est une clef de la théorie grammaticale de l'hébreu. D'une part, les formes des modes, temps et personnes du verbe sont ajoutées à la racine au moyen d'une série d'affixes. D'autre part, s'il se trouve que certaines consonnes de la racine sont «faibles», l'ajout d'affixes provoque des altérations prévisibles. Si l'on connaît les affixes d'une part, les règles d'altération de l'autre, on peut construire toutes les formes de tous les verbes quoique l'inverse soit plus délicat. C'est ce que nous appelons aujourd'hui à l'anglaise un model: un schéma théorique qui rend compte d'exceptions apparentes.

Par exemple, 'j'ai donné' est נתתי NTTY natatti et 'je donnerai' est אתן, 'TN etten; l'étudiant sait qu'au passé l'affixe personnel est תי -TY - ti (suffixé) et qu'au futur c'est $\aleph-e$ - (préfixé), de sorte que s'il ôte ces affixes il se trouve devant deux 'thèmes' différents, -natat- et -tten- (graphiquement נת et) alors qu'il devrait aboutir à un seul avec le sens de 'donner'. La solution élégante est que la 'racine' est נתTן NTN (attestée au passé 3 e singulier), soit natan, et que c'est la «faiblesse » du $\mathrm{N}$ final qui le fait s'assimiler au $\mathrm{T}$ du suffixe (natan $+t i>$ natatti) et

6 Voir l'édition de Jean Lallot à l'index des notions sous 'thème'. Occurrences en I, 76, avec note 185 ; II, 14, 18, 20 (avec note 36, voir aussi 37), 34, 131 (et note 66). 
la « faiblesse » du N initial qui, une fois que l'aphérèse de la $1^{\text {re }}$ voyelle (à cause de l'ajout du préfixe) met le $\mathrm{N}$ radical en contact avec le $\mathrm{T}$ radical, assimile le premier au second $(e+n a t a n>$ entan $>$ ettan puis etten). Le résultat est donc que, malgré les apparences, le verbe 'donner' a lui aussi une base ou racine trilittère unique, comme le veut la théorie.

\subsection{L'affixe}

Dans la Somme latine qu'il a écrite à partir des ouvrages d'Elie Levita, le Mele'ket ha-diqduq ha-šalem ou Opus grammaticum consummatum (1542, 1544, 1549, 1556), Sebastian Münster explique que Reuchlin a été le premier à utiliser le mot affixus (p. 136):

Diximus supra, Hebraeos duplicia habere pronomina, separata et affixa. De separatis iam diximus, de affixis autem nunc dicemus. Vocant ea Hebraei hakkinnuyim id est connotationes. Reuchlinus vero primus appellavit ea affixa. Nam subiunguntur, et affiguntur sic nominibus et verbis, ut in unam cum illis coeant dictionem $[\ldots]$.

«Nous avons dit plus haut que les Hébreux ont deux sortes de pronoms, les séparés et les affixes. Nous avons déjà parlé des séparés, parlons maintenant des affixes. Les Hébreux les appellent hak-kinnuyim, c'est-à-dire surnoms. Reuchlin a été le premier à les nommer affixes. Ils sont en effet joints ou fixés aux noms et aux verbes, au point de ne former avec eux qu'un seul mot [...].»

Sebastian Münster rend hommage à ses sources. Dans son Dictionarium hebraicum (1548), il dit dès le titre qu'il s'est servi du Sep̄er ha-šorašim 'le Livre des racines' de David Qimhị ; dans la préface il rend hommage à plusieurs aînés, surtout Reuchlin ; à la fin du livre, il donne une liste des Autores hebraei quos in hoc nostro lexico maxime sequuti sumus 'des [27] auteurs hébreux que nous avons surtout suivis dans notre lexique', parmi lesquels on trouve la massore, plusieurs auteurs de targums et le Talmud de Babylone. La liste nomme trois personnages de la famille Qimhi de Narbonne, en Provence: Joseph, le père, et les deux frères Moïse et David. On y voit aussi le nom d'un autre savant, sapiens Rabi Abraham, qui et Aben Ezra dicitur 'le sage rabbin Abraham qu'on nomme aussi Aben Ezra'.

Reuchlin était un latiniste et un helléniste distingué. Quand il étudie l'hébreu, il le fait en comparant avec les autres langues qu'il connaît, et il utilise ces comparaisons quand il estime qu'elles peuvent être utiles à ses lecteurs. Il réagit volontiers sur les critères formels: la construction des mots. Il remarque que les pronoms possessifs, par exemple $-\underline{k} a$ à la $2^{\mathrm{e}}$ personne masculin singulier (sus 'cheval' : sus-ka 'ton cheval') ou compléments d'objet du verbe (ya'avdu-ka 'ils te serviront') sont attachés au mot qu'ils complètent, et se ressemblent dans les deux fonctions. C'est différent de ce qui se fait en allemand ou en latin, où l'on utilise des pronoms détachés. Il décide de les appeler des affixa 'des (mots) attachés'. 
Münster a raison: on ne trouve pas le mot affixus (encore moins les deux autres praefixus ou suffixus) avant Reuchlin. Dans ses De Rudimentis hebraicis (1506), après les questions d'écriture et de lecture, Reuchlin donne un dictionnaire, le premier du genre (si l'on met de côté le lexique de Pellikan) qui s'étend des pages 32 à $545^{7}$. Arrive ensuite, au début du livre III, la Grammaire, p. 546-621. En voici le plan.

Illustration 1. Plan de la $3^{\mathrm{e}}$ partie des Rudimenta de Reuchlin: la Grammaire.

\begin{tabular}{l|l}
546 & Liber III Rudimentorum hebraicorum \\
\hline 550 & De oratione et eius partibus \\
\hline 551 & Partes orationis \\
\hline 552 & De Nomine et eius generibus \\
\hline & De Declinatione \\
\hline & (déclinaison des masculins) 556 \\
\hline & (des féminins) 560 \\
\hline & numeralia nomina 564 \\
\hline 585 & indeclinabilia 568 (et mots irréguliers) \\
\hline & (les 'articles' pour la déclinaison) 574 \\
\hline & pronomina quae demonstrant personas 575 \\
\hline & De Verbo \\
\hline & (les quatre conjugationes paal, piél, hip̄îl, hitpael) 585-6 \\
\hline & praesentis temporis 588 temporis 586 \\
\hline & futuri temporis 588 \\
\hline & verbum gerundivum quo sit infinitivus 589 \\
\hline & secunda conjugatio piqqed 593 \\
\hline & tertia coniugatio hip qid 594 \\
\hline & quarta coniugatio hitpaqqad 596 \\
\hline & defectiva (verbes) 599 \\
\hline & verbum grave plurium literarum 609 \\
\hline & de constructione verborum \\
\hline & de consignificativo ${ }^{8}$ \\
\hline & finis. \\
\hline & \\
\hline &
\end{tabular}

7 Sous la lettre aleph, la $1^{\text {re }}$ de l'alphabet, Reuchlin a 308 entrées, Pellican 61. C'est Pellican qui avait été le secrétaire de Reuchlin pour le début de dictionnaire. 
Le mot affixus intervient d'abord (p. 557) au moment où Reuchlin explique la déclinaison des noms. Il vient de donner des exemples et il résume :

Huius itaque declinationis primae communis est regula, ut cuiuscumque terminationis nomen masculinum numeri singularis tibi propositum fuerit, simpliciter ei cata paragogen addas iod et mem quod per hirek sonabit im. quod si regat post se casum, aut ei cohaereat pronomen subjunctivum, quod dicimus affixum, deponit mem finale.

«La règle commune de cette $1^{\text {re }}$ déclinaison [celle des noms masculins] est que, si tu trouves un nom masculin singulier de quelque terminaison que ce soit, tu lui ajoutes simplement kata paragôgên ${ }^{9}$ iod et mem [les deux lettres yod et mem], ce qui va se prononcer 'im' grâce à un hirek [hireq, un point souscrit]. Et si ce nom a un complément après lui, ou que s'y colle un pronom sub-joint ${ }^{10}$ (que nous appelons affixe), alors il perd son mem final. »

Ut autem quid dicam de regimine et affixis intelligas... 'Pour que tu comprennes ce que je vais dire du régime et des affixes, poursuit Reuchlin, il faut savoir qu'un nom peut avoir une forme différente quand il est in regimine, hoc est quod regat post se aliud nomen 'à l'état construit, c'est-à-dire quand il régit après lui un autre nom'. Notons que l'expression de regimen 'régime' qu'utilise Reuchlin est embarrassante en français, puisque ce que nous appelons un 'régime' ou 'cas régime' est en général le complément d'un autre mot, ce qui est régi par un autre mot (et que nous appelons le mot régissant) - alors qu'ici c'est l'inverse : le mot in regimine $^{11}$ est celui qui régit le mot qui vient ensuite, et c'est pourquoi les grammairiens en latin préfèrent souvent utiliser l'expression de status constructus 'd'état construit'. Dans les langues sémitiques c'est le terme régissant qui vient le premier, et c'est lui qui se modifie s'il a un complément. Ce complément peut être un pronom affixé : car là aussi, le nom régissant prend la forme in regimine 'à l'état construit'. Reuchlin associe ces deux situations.

Reuchlin sait que ce terme affixus 'affixe' est nouveau, et il en répète la description savante par pronomina subiunctiva 'pronoms sub-joints' à plusieurs reprises. Mais il utilise son terme nouveau avec constance et cohérence, toujours dans le sens de 'suffixe personnel', notamment dans les pages 559-560, 577 et 579583 où il en détaille la morphologie avec des exemples.

Dans le chapitre De constructione verborum, Reuchlin montre finalement (p. 610-612) comment construire les pronoms compléments de verbes, comme cidessous, où l'on voit qu'il s'agit des mêmes formes ${ }^{12}$ que pour les possessifs suffixés au nom.

9 Expression grecque: 'par dérivation'.

10 Par ce composé, Reuchlin entend 'joint après' - ce que nous appellerions un suffixe (sub-fixus).

11 Ailleurs aussi in statu regiminis, e.g. p. 562 : In statu vero regiminis, quando post se genitivum regunt.... Cette expression s'oppose à in statu absoluto, e.g. p. 564.

12 Sauf pour la $1^{\text {re }}$ du singulier. 
Illustration 2. Les affixes personnels en fonction d'objet, selon Reuchlin (p. 610).

\begin{tabular}{|c|c|}
\hline$p e^{‘} a l-o$ & il l'a fait \\
\hline pe'ala-hu & il l'a fait \\
\hline péalka & il t'a fait \\
\hline pe'ala-nî & il m'a fait \\
\hline pe'al-am & il les a faits \\
\hline pe'alkèm & il vous a faits \\
\hline pe'ala-nu & il nous a faits \\
\hline$p e^{c} a l-a h$ & il l'a faite \\
\hline pe'al-ek & il t'a faite \\
\hline pe'al-an & il les a faites \\
\hline pe'al-ken & il vous a faites \\
\hline
\end{tabular}

Ce qui lui fait conclure (p.613):

Visa itaque constructione affixorum tam in nominibus quam in verbis, oportebit te saepe et multum legere in biblia hebraica, ut inde alias constructiones quae variae sunt addiscas.

«Maintenant qu'on a vu la construction des affixes avec les noms et avec les verbes, il faudra que tu lises la Bible hébraïque souvent, et beaucoup, pour apprendre à partir de là les autres constructions qui diffèrent. »

Notons que la nécessité de s'entraîner change le statut de la Bible. On aurait pu croire que tout cet enseignement avait pour but de lire la Bible « dans le texte», mais ici on dirait que la Bible est devenue un champ d'exercices pour pratiquer la langue.

\subsection{Pagnino et la question des pronoms}

Le De Rudimentis hebraicis de Reuchlin (1506) était loin d'être rudimentaire, et faisait plus de 600 pages ; mais l'ouvrage comprenait 500 pages de dictionnaire, et on peut concevoir ces Rudimenta comme un manuel bien fait. Reuchlin ne souhaitait pas tout dire. Son livre comportait une quinzaine de pages sur l'écriture qui permettaient d'aborder le dictionnaire, et se terminait sur la grammaire (p. 545621). Les ouvrages monumentaux de Sante Pagnino (1470-1541) ont un caractère différent. Sa grammaire, les Institutiones, fait 421 pages (éd. 1526, qu'on va suivre ici ; l'éd. 1549 a 516 p. ${ }^{13}$ ), et il passe en revue tous les cas possibles jusque dans le détail. 
Sante Pagnino dépend assez peu, en apparence du moins, de Reuchlin et de sa terminologie. C'est probablement délibéré. Pour ce qui nous concerne, il retient la notion de regimen pour le nom suivi d'un complément, et appelle toutes les formes personnelles, indépendantes ou non, des 'pronoms', pronomina. Il connaît le mot affixus, rare chez lui, mais il l'emploie comme adjectif, lui donnant ainsi un statut plus descriptif que normatif :

II. 15. De coniunctione nominum cum pronomine (p. 102). Est et altera nominis coniunctio cum pronomine sibi affixo, quod relationem aut possessionem significat. Et exempli causa, sumatur unum nomen, et sit davar verbum, quod in regimine genitivi facit devar. Et cum pronomine devar-o verbum eius, verbum suum.

«Conjonction des noms avec un pronom. Il y a une autre conjonction du nom, avec un pronom qui lui est affixé, qui signifie une relation ou une possession. Et par exemple, prenons un nom, disons davar 'parole', qui au régime de génitif fait devar. Et avec un pronom devar-o 'sa parole'."

Il utilise le mot coniunctio pour désigner le fait syntaxique, manifesté au plan morphologique et phonologique par un changement vocalique dans le premier des deux mots liés - que le second soit un nom ou un pronom.

Il s'en explique dans son chapitre sur les pronoms (II.34, p. 201-206): 'Le pronom: ce que c'est, d'où vient son nom, ce qu'il signifie, et ses différentes sortes'. Pagnino explique qu'il existe deux sortes de pronoms, le pronom 'conjoint et affixé aux noms et aux verbes', qui signifie la relation ou la possession, et le pronom séparé qui est généralement un démonstratif; car c'est dans les démonstratifs qu'il range les pronoms personnels indépendants. Voici le début de sa description (p. 202):

Est enim pronomen nismak coniunctum et affixum nominibus (ut dictum est supra capite XV) et uerbis, ut suo dicemus in loco. Et nominibus iunctum aut qinyan possessionem, aut șirup ${ }^{14}$ relationem significat. Sunt quippe pronomina possessiva, ut meus, tuus, suus, noster et vester, et relativa, ut ipse, ipsa, ipsum, ille, illa, illud. Verbis vero affixum, aut patientem aut agentem personam indicant, patientem ut psalmo 30,2 aromim-ka yehovah ki dillita-ni. Exaltabo te Domine, quoniam exaltasti me, et id genus. Agentem vero, ut in plerisque hammecoroth, id est infinitiuis et gerundiis, ut 2 Reg. 6, 21, ki-re'ot-o 'ot-am cum vidisset ipse eos, et idgenus, de quibus suo loco dicturi sumus.

«Il y a donc le pronom nismak $k^{15}$, c'est-à-dire conjoint et affixé aux noms (comme on a dit plus haut en II.15) et aux verbes (comme on dira plus loin). Joint aux noms, il signifie qinyan 'la possession', ou șirup 'la relation'. Ce sont les possessifs comme 'mon, ton, son, notre, votre', ou relatifs comme 'il, elle'. Mais affixés aux verbes, ils indiquent la personne agent ou patient. Patient, comme au Ps 30, 2 aromim-ka yehovah ki dillita- $n i$ 'Je t'exalterai Seigneur car tu m'as

14 Le terme exact serait șerup.

15 Sur cette racine et les emplois de semîxah ou semîxût pour 'état d'annexion', Prijs 1950, p. 85. 
exalté', etc. Agent en revanche, comme dans la plupart des hammecoroth [hameqorot], c'est-à-dire les infinitifs et gérondifs, comme 2 Rois, 6, 21, ki-re'ot-o 'ot-am 'comme il les avait vus' ${ }^{16}$, etc. et on en parlera plus loin. »

Alterum pronomen est nip̄rad separatum a nomine et verbo, quod dividitur in activum et passivum. Et horum quaedam sunt demonstrativa, ut ani Ego communis generis $[\ldots]$.

«L'autre pronom est nip̄rad 'séparé' du nom et du verbe ${ }^{17}$. On le divise en actif et passif. Certains sont démonstratifs, comme ani 'je, moi' à tous les genres $[\ldots] . »$

Dans son livre sur Les Etudes hébrä̈ques en France ${ }^{18}$ Sophie Kessler-Mesguish notait que « Le terme d'affixe, employé d'abord par Reuchlin, est repris par la quasitotalité des grammairiens de notre corpus » delà de la France, puisque Pagnino est réticent, et d'autre part le partage avec pronomen n'est pas forcément celui qu'on attendrait. Quand Pagnino appelle pronomen un affixe personnel, nominal ou verbal, il a raison - mais évidemment il se place au plan sémantique, tandis que la terminologie de Reuchlin vise la morphologie.

On observe des emplois flottants de ce vocabulaire nouveau. Dans les éditions ulérieures de la Tabula de Clenard ${ }^{19}$, on trouve l'étrange expression de inseparatus 'non-séparé' pour les affixes. Voici son texte, où l'on va retrouver des éléments bien connus (p. 80-81 20 ):

Cur puncta mutentur. Fontes unde oritur vocalium permutatio sunt quatuor, Regimen, Affixum, Pluralis numerus, Genus foemininum. Regimen est quando dictio ipsa regit aliam, quod Hebraei vocant samuk samuch, id est continuum, coniunctum, sive vicinum, ut debar ab $\underline{i}$ verbum patris mei. Hîc, alia puncta quam cum absolute, ac citra regimen dicimus davar verbum, id quod illi appellitant mukeret $^{21}$ mucheret, hoc est absolutum dictionis statum. [...] Affixi voce pronomina quaedam intellige hoc donata vocabulo, quod numquam inueniantur separatim, sed perpetuo ad finem dictionis adhaerescant, ut debaro verbum eius etc.

Pronomina inseparata, quae et affixa a situ suo dicuntur. [Suit le tableau des suffixes]

16 Les formes nominales du verbe, ici reot- 'fait de voir, vision' peuvent recevoir un affixe possessif marquant alors le sujet-agent reot-ô 'sa vision' (= le fait qu'il voit), et par ailleurs ici un complément d'objet introduit par une préposition ôt-am : kî-reot-ô ôt-am 'du fait de sa vision d'eux' (= quand il les vit).

17 Ibn Ezra utilise ha-nifradîm pour les mots sans suffixes (Prijs 1950, p. 113).

18 Kessler 2013, p. 204. Ce livre, posthume, repose sur son travail de thèse. La thèse de Sophie Kessler-Mesguich (1957-2009), a été soutenue en 1994.

19 Y compris celles qui sont refondues par Jean Cinqarbres à partir de 1550, ainsi dans celle de 1564 qui est intéressante.

20 Dans les éditions plus anciennes, avant 1550, ce texte est p. 112-113, mais le tableau n'y est pas, ni l'expression 'inseparata'.

21 À cet endroit se place une note de Genebrard: Lege mukerat quasi excisum, a regimine scilicet, etc. Genebrard corrige Clénard: mukerat est le terme exact. 
«Pourquoi les points [les voyelles] changent. Les sources du changement vocalique sont quatre : le régime, l'affixe, le pluriel, le féminin. Le régime, c'est quand un mot en régit un autre, ce que les Hébreux appellent samuk 'continu, conjoint, voisin', comme dans debar abi 'parole de mon père'. Là, on a une ponctuation autre qu'à l'absolu, et hors du régime on dit davar 'parole', et c'est ce qu'ils appellent mukeret, l'état absolu du mot. [...] Les pronoms dits affixes sont désignés par ce terme parce que on ne les trouve jamais séparément, mais ils collent toujours à la fin du mot, comme dans debaro [devar-o] 'sa parole' etc.

[Titre du tableau qui suit:] Pronoms inséparés, dit aussi affixes à cause de leur place. »

Mais si on les appelle Pronomina inseparata, c'est pour les différencier de ceux qu'on ne découvrira qu'à la fin de l'ouvrage, les Pronomina separata: nos pronoms indépendants. Ajoutons que ces éditions successives et influentes de la Tabula de Clenard utilisent affixa aussi pour les indices personnels compléments des verbes, dans la tradition de Reuchlin.

\subsection{Richard du Mans commente les traductions des psaumes, 1541}

En 1541 paraît à Paris une nouvelle édition du Commentaire des psaumes de Pierre Lombard. Le commentaire de Richard du Mans ${ }^{22}$, qui fait l'originalité de cette édition $^{23}$, peut servir à mesurer la présence du nouveau lexique. Richard du Mans est un hébraïsant, et l'essentiel de son propre commentaire consiste à comparer les nombreuses traductions qui ont été faites des psaumes. Il cite la Septante et Jérôme, mais aussi les traductions ou études récentes de Münster, Pagnino, Pellikan, le Psalterium quintuplex (1509 et 1515) de Lefèvre d'Etaples, et plusieurs autres. Il cite aussi 'David Kimhi' une douzaine de fois, souvent à travers Münster, et le Liber radicum est mentionné à plusieurs reprises.

Richard utilise, dans leur sens grammatical, à la fois radix et thema, mais le premier mot (95 fois) plus que le second (41 fois). Les deux semblent avoir chez lui exactement le même sens; l'expression a radice $X$ peut être souvent employée directement dans des énoncés comme 'tel mot est de la racine $X$ ', mais aussi avec une intéressante diversité de verbes : venire, ducere, deducere, derivare, deflectare et même descendere. Il utilise volontiers les deux mots 'racine' et 'thème' dans la même notice, par exemple (sur le Ps. 9, 21. PL 191, 145B-C) :

22 Ce personnage a participé à l'élaboration de la Vulgate révisée tridentine. Voir Höpfel 1908, Allgeier 1951.

23 L'ouvrage est accessible en ligne dans l'édition originale (voir in fine), et a été repris entier dans la Patrologie, PL 191. Nos recherches lexicographiques ont été rendues possibles par le moteur de recherche Chadwyck sur la PL, qui est accessible grâce à la Bibliothèque Interuniversitaire de la Sorbonne. 
Hoc provenit ideo quod vocabulum morah potest deflecti a radice iare cum aleph in fine, significabitque terrorem: Vel a themate iarah per he in fine, id est docuit, ut morah seu moreh sit doctor, seu legislator. Aleph autem et he, apud Hebraeos, sunt litterae passim commutabiles.

«Cela vient du fait que le mot morah peut provenir de la racine YR, avec un aleph à la fin, et il signifiera alors terreur, ou bien du thème YRH avec un $\mathrm{H}$ à la fin, 'enseigner', de sorte que morah ou moreh signifie 'docteur, législateur'. C'est que l'aleph et le he sont des lettres assez interchangeables chez les Hébreux. »

Que ce soit avec thema ou, plus souvent, avec radix, l'exercice d'identification de la racine est très fréquent dans son commentaire, parce que c'en est la nouveauté. Cela lui permet d'expliquer les traductions différentes, et il donne son avis sur la meilleure; parfois, il ne tranche pas. Il utilise non seulement radix, mais aussi litera radicalis et aussi le terme nominalisé au neutre de radicale; il emploie aussi affixum. Il a une position assez prudente, à mi-chemin entre Reuchlin et Pagnino: souvent affixum pronomen ou pronomen affixum (324D, 562C, 1010D), affixum seu pronomen (93B, 366D) 'affixe ou encore pronom'. Il reste deux passages où il est plus audacieux et parle de littera affixa. Il explique que le mem final $-\mathrm{M}$ d'une forme verbale discutée doit s'ajouter:

rodem, si ducatur a radice radah et littera mem sit affixa, significat dominari. (Sur Ps. 68, 28 ${ }^{24}$ )

«(la forme) rodem, si on la tire de la racine radah et que la lettre mem est affixe, signifie 'dominer'.»

Le terme d' 'affixe' conserve bien le sens spécifique d'indice personnel suffixé que lui avait donné Reuchlin ; mais il tend à désigner une opération morphologique plutôt qu'une classe de mots.

\subsection{Buxtorf: préfixe et suffixe}

Quand Buxtorf est étudiant à Herborn en 1585, l'hébreu est déjà au cursus ordinaire des étudiants $^{25}$. Sans doute était-ce en pays de Réforme, mais les catholiques ne veulent pas être en reste: la science de l'hébreu, nous en donnerons un exemple plus loin, est une arme dans un combat idéologique.

Jean Buxtorf (l'Ancien : 1564-1629, par rapport à son fils qui portait le même nom : 1599-1664) a donné à la théorie morphologique une impulsion décisive en transformant la portée de la notion d'affixe. Buxtorf écrivit deux sortes d'ouvrages de grammaire : des manuels, dont l'Epitome souvent réimprimé, et un ouvrage de fond, le Thesaurus grammaticus. Ce dernier, dans la $5^{\mathrm{e}}$ édition de 1651 , publiée à Bâle, s'étend sur 734 pages. Voici un sommaire: 
Illustration 3. Le plan du Thesaurus grammaticus de Buxtorf

\begin{tabular}{|c|c|c|}
\hline 1.1 & De literis & 1 \\
\hline 1.2 & De vocalibus et diphthongis & 8 \\
\hline 1.3 & De dages et mappik & 12 \\
\hline 1.4 & De syllaba et legendi ratione & 14 \\
\hline 1.5 & De accentibus & 28 \\
\hline 1.6 & De notatione vocum [vox signifie 'mot' chez Buxtorf] & 64 \\
\hline 1.7 & De divisione vocis in genera et species & 66 \\
\hline 1.8 & De nomine & 67 \\
\hline 1.9 & De prima declinatione nominum & 77 \\
\hline 1.10 & De secunda declinatione & 83 \\
\hline 1.11 & De pronomine & 87 \\
\hline 1.12 & De verbo & 91 \\
\hline $1.13-20$ & De conjugationibus verborum $(\ldots)$ & 98 \\
\hline 1.56 & De nominibus verbalibus & 296 \\
\hline 1.57 & De voce indeclinabili, et primum de adverbio & 325 \\
\hline 1.58 & De praepositione & 328 \\
\hline 1.59 & De conjunctione & 330 \\
\hline 1.60 & De interjectione & 331 \\
\hline 2.1 et 2 & De convenientia nominis cum nomine & 333 \\
\hline 2.3 et 4 & De syntaxi nominum quâ unum regit alterum & 356 \\
\hline 2.5 & De syntaxi pronominum cum nominibus & 382 \\
\hline 2.6 & De syntaxi pronominis separati cum nomine & 388 \\
\hline 2.7 et 8 & De syntaxi prnominum nominibus a fine affixorum & 395 \\
\hline 2.9 et 10 & De convenientia verbi cum nomine & 421 \\
\hline 2.11 & De syntaxi verbi cum dativo $(\ldots)$ & 445 \\
\hline 2.14 & De syntaxi verbi substantivi hayah. & 488 \\
\hline 2.15 & De syntaxi verborum passivorum & 495 \\
\hline 2.16 & De syntaxi verbi infinitivi & 496 \\
\hline 2.17 & De syntaxi verbi cum pronominibus inseparabilibus & 502 \\
\hline 2.18 & De syntaxi participiorum & 529 \\
\hline 2.19 & De syntaxi adverbiorum & 535 \\
\hline 2.20 & De syntaxi praepositionum & 553 \\
\hline 2.21 & De syntaxi conjunctionum & 580 \\
\hline 2.22 & De syntaxi interjectionum & 596 \\
\hline 2.23 & De distinctionibus sententiarum per accentus & 598 \\
\hline
\end{tabular}


À propos des diverses sortes de pronoms, en 1.11, il écrit ceci (p. 84-89 - texte de la $6^{\mathrm{e}}$ éd.) :

Pronomen est, quod vice Nominis ponitur. Est enim secundarium quoddam Nomen, occulte Nomen antecedens referens. Hinc Pronomina Hebraeis dicuntur kinnuyim Cognomina, quod Nominis principalis sint aliud quoddam cognomen. [...] Pronomina sunt vel Separata (Hebr. nip̄radim), vel aliis vocibus Conjuncta (Hebr. mehubbarim), sive Separabilia et Inseparabilia. Separata, quae separatam dictionem constituunt; suntque vel Integra vel Defectiva. Integra, quae genere et numero distinguuntur: suntque tria, tres personas indicantia ${ }^{26} .[\ldots]$

«Un pronom est ce qu'on met à la place d'un nom. C'est une sorte de nom secondaire, qui réfère en secret à un nom qui précède. C'est pourquoi en hébreu on les appelle kinnuyim 'surnoms', parce qu'ils sont une façon de surnom du nom principal. [...] Les pronoms sont ou séparés (héb. nip̄radim) ou conjoints à d'autres mots (mehubbarim), en d'autres termes séparables et inséparables. Les [pronoms] séparés constituent un mot à part. Ils sont complets ou défectifs. Les complets sont ceux qui distinguent genre et nombre : il y en a trois, qui indiquent les trois personnes. $[\ldots] »$

Defectiva sunt, quae genere vel numero deficiunt.

«Les défectifs sont ceux qui sont incomplets ${ }^{27}$ pour le genre ou le nombre.»

Il s'agit des pronoms-adjectifs démonstratifs. Puis viennent le pronom relatif et les interrogatifs. La nomenclature annoncée reprend ensuite (p. 87).

Conjuncta allis vocibus sunt, vel Praefixa vel Suffixa.

«Les conjoints, le sont à d'autres mots, soit préfixés soit suffixés.»

Praefixa duo sunt, utriusque generis et numeri, še- et $h a-$, sequente post utrumque dagesch ${ }^{28}$. Illud ex ašer desumptum est et relativè significat, ut ipsum ašer; hoc ex hu, et nunc Demonstrativum est, Hic, haec, hoc, nunc relativum qui, quae, quod. Priori modo frequentissime ante Nomina usurpatur, tanquam articulus communis omnium casuum $[\ldots]$.

«Les préfixés sont deux, indifférents au genre et au nombre ${ }^{29}: \check{s} e$ - et $h a-$, tous deux suivis d'un dagesh. Le premier vient de ašer et a la signification d'un relatif, comme $a \check{s} e r$ lui-même. Le second vient de $h u^{\prime}$, et est tantôt démonstratif (hic, haec, hoc) et tantôt relatif (qui, quae, quod). Dans le premier sens [démonstratif] il est très souvent utilisé devant les noms, comme un article, valant pour tous les cas $[\ldots]$.»

Suffixa sunt, quae aliis vocibus a fine affiguntur, et in specie vulgo affixa vocantur. Oriuntur tamen ex integris, hoc modo.

$26 \mathrm{~S}$ 'insère à cet endroit un tableau des pronoms indépendants, puis un examen de chacune des formes du tableau.

27 C'est-à-dire qui n'offrent pas les distinctions de genre ou de nombre.

28 Il s'agit d'un point qui signale le redoublement de la consonne.

29 Ces deux préfixes, ou proclitiques, sont invariables. Le premier est un relatif, le second un article. 
«Les suffixés sont ceux qui se fixent à la fin des mots : ce sont ceux qu'on appelle 'affixes' d'habitude. Ils proviennent cependant de pronoms pleins. ${ }^{30}$

C'est « l'installation officielle» des mots préfixe et suffixe en langue latine ${ }^{31}$. Ils auront une longue carrière en latin technique, et s'installeront dans les langues d'Europe soucieuses de se forger un lexique savant.

\section{Sur lE SORT ULTÉRIEUR DES MOTS 'RACINE', 'AFFIXE', 'PRÉFIXE', 'SUFFIXE'.}

\subsection{Une querelle savante}

Une des premières controverses savantes en français où intervient le mot préfixe aidera à comprendre pourquoi tant de gens, hors des communautés juives, voulaient savoir l'hébreu; et pourquoi le lexique technique forgé avec la philologie hébraïque a pu se répandre au-delà de son creuset d'origine.

\section{D ISSER T A T ION. 277} que vous voulez ćxempter de falfification.

J'ai éré un peu plus furpris, je vous l'avouë, que vous ayez voulu juftifier ce mor même, que votre Traducteur a ajôtté à l'Hebreu, puis qu'il n'eft exprimé ni ablorbé dans la contraction, d'où. vous prétendez le déterrer, pour lui faire voir le jour. Quand on accordetoit que la préfixe ה, répond à 'article des Grecs, $\dot{0}, \dot{\eta}, \boldsymbol{i}, \&$ ì l'article srançois, le, la, je ne vois pas quel avantage vous en pourriez tirer, pour conclure autre chofe, fitson que bammikra répondroit à l'y ávázwwas des Grecs; lectio, des Latins; \& la letture, dans notre Langue, ou felon vous, l'écriture. Ce n'eft douc pas fans raifon qu'on s'eft fcandalifé du mot meme, \& qu'on a dit qu'il eft inféré pour rendre la falfification complette.

Illustration 4. Le texte du Jésuite d'Alençon où il utilise «la préfixe ה».

Le texte ci-dessus, et l'essai de Pierre-Daniel Huet (1630-1721) qui y répond, figurent dans un recueil de $1720^{32}$. Le texte de Huet est un arbitrage dans une querelle entre catholiques et protestants. Un ministre protestant avait utilisé un

30 Suit un tableau des suffixes personnels, face aux pronoms indépendants correspondants.

31 Notons que Buxtorf n'utilise pas ce vocabulaire (affixe, préfixe, suffixe) pour les préfixes ou suffixes personnels marquant le sujet des verbes (et formant donc les conjugaisons). Il dit par exemple pour le passé (p. 95) Persona est specialis terminatio verbi 'la personne est une terminaison spécifique du verbe' Et pour le futur (p. 96) Formantur autem ejus personae ab Imperativo, assumptis ab initio quatuor literis characteristicis 'On en forme les personnes à partir de l'impératif, en plaçant au début quatre lettres spéciales'.

32 Dissertations sur différens sujets, composées par M. Huet ancien évêque d'Avranches et par quelques autres savans (1714 ?, La Haye 1720). L'abbé de Tilladet, éditeur du volume, raconte dans sa Préface les circonstances qui ont amené Huet à rédiger cet essai philologique. 
passage du livre biblique de Néhémie $(8,8)$ pour montrer que le croyant n'a pas besoin des commentaires de l'Église, et peut comprendre les textes par lui-même. C'est une revendication classique de la Réforme. Huet, consulté, aboutit à la paraphrase suivante du verset (p. 285) : «Esdras et ses compagnons lurent dans le livre de la Loi de Dieu, qui était traduit et expliqué. Et le peuple écouta attentivement, et avec application d'esprit, et il entendit ce qu'on lisait». Il commente ensuite les points principaux débattus dans la correspondance entre le ministre et le jésuite, et en vient (p. 286) à la question de l'article (orthographe modernisée) :

Pour justifier la version de bammikra ${ }^{33}$, il [le ministre] dit que la préfixe ה répond

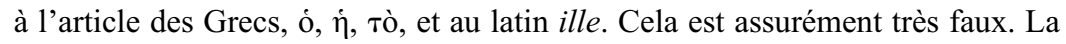
préfixe $ה$, et l'article des Grecs, sont souvent oiseux; et l'ille des Latins ne l'est jamais. [...] Dans ce chapitre même vous y trouverez souvent ces mots répétés כל העם, que les Septante ont fort bien traduit $\pi \tilde{\alpha} \varsigma$ ó $\lambda \alpha$ òs, et que les versions françaises traduisent bien aussi, tout le peuple, et le latin omnis populus et non pas omnis ille populus, ni tout ce peuple. ${ }^{34}$

Il est clair entre ces savants messieurs, quand bien même ils ne sont pas d'accord, que « la préfixe ha» est une expression limpide : tout le monde sait ce que c'est. Le mot est acquis. Mais c'est vrai qu'il s'agit de l'analyse d'une phrase en hébreu. D'autre part, si le mot préfixe est encore adjectif, puisque le féminin est dû au fait que le mot lettre est sous-entendu, il n'en est pas moins un adjectif substantivé : il est tout près d'acquérir son indépendance. Et ce vocabulaire n'est pas toujours confiné à l'hébreu. Dans la présentation que l'éditeur de 1720 fait d'un autre essai de Huet, on trouve ${ }^{35}$ (orthographe modernisée) :

L'étroite liaison qui a été entre Monsieur l'Évêque d'Avranches [Huet] et feu Monsieur Ménage $^{36}$, depuis la jeunesse du premier jusqu'à la mort du second, donnait au savant prélat la liberté de lui expliquer nettement ses sentiments touchant ses ouvrages, dont les Étymologies de notre langue ont fait le principal. Il lui remontrait souvent qu'il abusait de son talent, et que la grande connaissance qu'il s'était acquise par un long usage de la permutation des lettres, lui faisait

33 Les traductions protestantes considèrent que le dernier mot du verset (que Huet transcrit bammikra et va interpréter "ce qu'on lisait») désigne la Bible elle-même, ainsi dans la version d'Ostervald «Et ils lisaient au livre de la loi de Dieu, et ils l'exposaient et en donnaient l'intelligence, la faisant entendre par l'écriture même. » (le dernier mot est en italique dans les Bibles protestantes, pour montrer que c'est un ajout destiné à faciliter la compréhension). En hébreu, ce dernier mot contient le mot miqra' précédé de l'article (qui provoque le redoublement du $m$ ) et de la préposition be- 'dans'.

34 Dans l'hébreu kol ha-'am (tout le-peuple) on a l'article ha-préfixé au nom 'am 'peuple'. Dans le grec pas ho laos on a aussi un article ho devant laos 'peuple'. Mais en latin où il n'y a pas d'article, on dit seulement omnis populus : ille n'est pas un article.

35 Préface, dissertation 19.

36 Il s'agit de Gilles Ménage (1613-1692), l'auteur des Origines de la langue française, 1650, considérées comme le premier dictionnaire étymologique du français. 
quelquefois mépriser les règles fondamentales de son art, dont une des principales est de ne chercher l'origine des mots dérivés que dans les lettres radicales des primitifs, et non dans les serviles, qu'autrement on pourrait dériver [...] lentus de somnolentus [lent de somnolent], et leo d'equuleus [lion de poulain].

\subsection{Deux exemples : affixe et préfixe}

Le terme affixe, qu'avait lancé Reuchlin en 1506, a été le premier à trouver une pratique en langue française. Il se trouve en français chez André Thévet à la fin du siècle, en 1584, dans ses Pourtraits et Vies des Hommes illustres. C'est dans son éloge de Sebastian Münster, cosmographe comme lui ${ }^{37}$ :

Premièrement je produirai l'introduction qu'il a fait [sic] pour la langue hébraïque, avec une telle facilité, que les plus lourds peuvent, presque en dormant, comprendre les secrets d'une si sainte et divine langue. Vous avez des tables, grammaires déparcelées et jointes ensemble pour l'explication de toutes les parties de la langue hébraïque soit sur les déclinaisons des pronoms, les conjugaisons de verbes, l'artifice de soumettre [affixer] les affixes, les façons diverses et jugements des noms, les explications des consignificatifs [les particules], les abréviations magistrales, les difficultés des accents et la composition des vers.

Ce passage montre l'aisance des lecteurs de ce temps pour faire passer les mots latins en français (nous le savons par Rabelais, Montaigne ou Du Bellay). Il suggère à bon droit un rapprochement entre les cartes et les tableaux synoptiques. Thévet met l'accent, dans son éloge de Münster, sur les 'tables', 'grammaires déparcelées et jointes ensemble', c'est-à-dire sur sa capacité à nous donner à voir l'ensemble des faits pertinents, au lieu de les traiter par 'parcelles'. Il y a là un fait important de l'histoire des idées ou des représentations, car ce n'est pas une rencontre de hasard, dans l'esprit de Sebastian Münster, que celle de la visibilité d'un paysage complet dans la représentation graphique d'une carte, avec l'assemblage des faits de langue dans un tableau synoptique.

Dans l'Encyclopédie (1751), Dumarsais (à l'article «Article») utilise le mot préfixe:

En Hébreu, en Chaldéen, \& en Syriaque, les noms sont indéclinables, c'est-àdire, qu'ils ne varient point leur désinence ou dernieres syllabes, si ce n'est comme en François du singulier au pluriel ; mais les vûes de l'esprit ou relations que les Grecs \& les Latins font connoître par les terminaisons des noms, sont indiquées en Hébreu par des prépositifs qu'on appelle préfixes, \& qui sont liés aux noms, à la maniere des prépositions inséparables, ensorte qu'ils forment le même mot.

Comme ces prépositifs ne se mettent point au nominatif, \& que l'usage qu'on en fait n'est pas trop uniforme, les Hébraïsans les regardent plûtôt comme des 
prépositions que comme des articles $^{38}$. Nomina Hebraïca proprie loquendo sunt indeclinabilia. Quo ergo in casu accipienda sint \& efferenda, non terminatione dignoscitur, ut apud Latinos et Graecos, sed: Primo et proecipuè constructione, et loci exigentia, id est congruo orationis sensu, ut agnoscit ipse Guarinus, pag. 69. Secundo praepositionibus quibusdam, seu litteris praepositionum vices gerentibus, quae ipsis à fronte adjiciuntur. Masclef. Gramm. Hebr. c. II. n. 6.

\subsection{La généralisation de la notion d'affixe}

$\mathrm{Au}$ début du $\mathrm{XIX}^{\mathrm{e}}$ siècle, quand Frédéric Schlegel jette les bases de la grammaire comparée, le mot de 'racine' dans son sens technique est d'un emploi courant. Il publie en 1808 Über die Sprache und Weisheit der Indier. Ein Beitrag zur Begründung der Alterthumskunde. Voici un extrait du résumé qu'en fit son second traducteur en français, Mazure, en 1837 :

Il y avait deux points de vue divers pour établir l'affinité des langues d'une même famille: d'abord l'analogie de la racine, en suivant les séries de lettres faites pour s'échanger mutuellement, ou bien en marquant certaines mutations hors de règle et particulières à certaines langues; ensuite il fallait observer la structure grammaticale qui joue un si grand rôle et si peu contesté dans l'enchaînement et la formation des langues, soit que ces langues aient ou n'aient pas d'affixes, qu'elles aient ou n'aient pas de flexions, que leurs syntaxes soient plus ou moins régulières et analogiques, que leurs conjugaisons soient plus ou moins riches, complètes, nuancées, répondant à tous les besoins de la conception. Or, c'est là ce qui se trouve dans la première partie du livre de Schlegel.

Quand Mauget en 1809 avait une première fois traduit le début de l'ouvrage, il employait couramment le mot de racine (p. 114-115). On notera aussi le mot de structure:

L'ancienne langue de l'Inde, appelée par ses habitants Sanscrito [...] a la plus grande affinité avec les langues romaine et grecque, ainsi qu'avec les langues germanique et persanne. La ressemblance gît non seulement dans un grand nombre de racines ${ }^{39}$ mais encore elle s'étend jusqu'à la structure intime de ces langues.

38 La citation de Masclef est corrompue dans beaucoup de versions en ligne. Elle est rétablie ici depuis l'original: F. Masclef, Grammatica hebraica, II, 6 (p.51-52), ici l'édition Paris \& Bologne 1750. «Les noms hébreux, à proprement parler, sont indéclinables. Quel cas ils doivent recevoir et faire paraître, on ne le reconnaît pas à la terminaison comme en latin et en grec, mais: D'abord et surtout par la construction et le contexte, c'est-à-dire le sens satisfaisant du discours, comme Guarinus (page 69) le reconnaît lui-même. Ensuite par des prépositions, ou des lettres qui font office de prépositions et qui sont ajoutées à la tête des mots.» Il s'agit de Pierre Guarin (Petrus Guarinus), qui a publié en 1724 à Paris sa Grammatica hebraica et chaldaica, où il écrit en effet (p. 69b): Verum nominum hebraeorum casus longe melius ex structura sermonis et ratione syntactica distinguuntur, quam ex quatuor articulis [ha-, le-, èt-, mi-] supra memoratis.

39 On sait que la question de la communauté des racines dans des langues différentes se trouve déjà dans l'article «Langue (grammaire)» de Beauzée, dans l'Encyclopédie (article III, §1, $\left.1^{\circ}\right)$, vol 9, 1765 . 
C'est aussi dans cette traduction qu'on voit apparaitre le mot suffixe. Nous donnons ici des passages du chapitre 4, dans la traduction Mazure, qui est meilleure (p. 52-54, avec une coupure) : on y trouve les trois mots : affixe, préfixe, suffixe.

Les langues américaines forment leur grammaire par des affixes. C'est pourquoi elles sont, comme toutes les langues de cette classe, très-riches en rapports pronominaux, exprimés par des suffixes, et par conséquent riches en verbes et en conjugaisons relatives. [...]

Il est vrai que les particules ajoutées peuvent finir par s'absorber dans le mot principal, au point que l'on ne puisse les reconnaître, et qu'elles soient prises pour de véritables flexions. Mais si, dans une langue telle que l'arabe, par exemple, et toutes celles de la même famille, on voit que les rapports les plus essentiels et les premiers par leur importance, comme les rapports de personne dans les verbes, se rendent par l'addition de quelques particules significatives, et que l'emploi de suffixes de cette sorte semble appartenir au fond même de la langue, alors on admettra sans difficulté que la même chose s'est présentée dans d'autres cas où l'addition des particules étrangères ne se laisse pas si aisément reconnaître. Au moins, on peut conclure avec assurance que cette langue, vue dans sa généralité, appartient à la classe de celles qui emploient les affixes, bien que dans le détail, soit par l'effet d'un mélange, soit par un perfectionnement artificiel, elle ait déjà revêtu un caractère nouveau et plus parfait.

Dans le texte allemand de 1808, on trouve Affixa (8 fois), Präfixe (3 fois), Suffix (265 fois). ${ }^{40}$

\section{Perspectives}

La métaphore de la dénudation, d'ôter du mot les vêtements successifs que sont les affixes et diverses extensions pour trouver à la fin le corps nu de la racine, est de Reuchlin ${ }^{41}$.

Ex his omnibus per nos hactenus expositis facilius scies id, quod in lingua hebraica maximae dicitur artis esse. ac ingenii singularis laudatissimum indicium. cum omnis dictio aut primitiva sit aut derivativa. exuere nomen derivativum singulis vestibus ad nuditatem quousque appareat primitivum latine prius tecum agam. exempli gratia si hoc nomen pluralis numeri. hae inhonorificabilitudines velis ad primitivum statum redigere. quid facies? decorticabis illus omnibus tunicis quousque absolutum appendiciis appareat primitivum. sic. Reiice primo articulum hae. secundo compositionem primam qua ligatur cum praepositione in. tertio compositionem qua ligatur cum ifica. a facio. quarto terminationem verbalem aptitudinis quae est bilis. quinto

40 Le texte se trouve à http://www.deutschestextarchiv.de/book/show/schlegel_indier_1808, où l'on peut chercher toutes les occurrences. Pour Suffix, le moteur de recherche produit un schéma intéressant qui donne la fréquence du mot en fonction des années.

41 Rudimenta p. 582. Sophie Kessler-Mesguich (2013, p. 205 et note 24) avait déjà signalé ce passage. 
terminationem abstractionis quae est tudo. sexto casualem inflectionem genitivi quae est din. septimo numeralem terminationem es. separatis [583] igitur hae. in. ifica. bili. tudin. es. quid remanet? certe nudum illud quod nominamus honor. ecce primitivum. nunc pariter operare in hebraicis. ad cuius faciliorem aditum scire te iubeo.

«Avec tout ce que nous avons montré jusqu'ici, tu vas mieux comprendre ce qu'on regarde en hébreu comme du grand art, et le signe admirable d'un génie singulier, puisque chaque mot doit être ou primitif, ou dérivé : défaire un nom dérivé de chacun de ses vêtements jusqu'au corps nu, jusqu'à faire apparaître le nom primitif - je vais te le montrer d'abord en latin. Par exemple, si tu voulais retrouver l'état primitif de ce nom au pluriel hae inhonorificabilitudines 'ces inhonorificabilitudes', qu'est-ce que tu ferais? Tu vas le décortiquer de tous ses manteaux, jusqu'à ce qu'apparaisse, délivré de ces appendices, le mot primitif. Allons-y. Enlève (1) pour commencer l'article hae 'ces'. Ensuite (2) le premier degré de composition donné par la préposition in [négative]. Ensuite (3) le second degré avec - ifica, qui vient de facio 'faire'. (4) La terminaison verbale d'aptitude en -bilis. (5) La terminaison abstraite -tudo. (6) l'inflexion casuelle du génititif en -din. (7) la terminaison de nombre en -es. On a donc ôté hae, in, ifica, bili, tudin, es: qu'est-ce qui reste? En bien, tout nu, cela que nous nommons honor 'honneur' ; voilà le primitif. Maintenant, tu vas faire pareil en hébreu, ce qui est plus facile avec mon introduction!»

Il y a chez Reuchlin ce double mouvement que nous avons souligné plus haut. Sans doute, il y a quelque chose de fascinant dans ce dévoilement progressif des racines primitives, à une époque où l'on croyait généralement que l'hébreu était la langue de l'origine, celle d'Adam. Mais en même temps c'est une technique - et nous avons vu comment Reuchlin encourageait son élève à s'exercer sur ce «terrain» qu'est le texte biblique. Cette technique est faite de méthode et d'instruments, comme un métier, et ce découpage en tranches a quelque chose de rassurant: il faut couper, délicatement et dans le bon sens.

C'est pourquoi le terme d'affixe n'est pas neutre, et manifeste une décision critique. Bien sûr, comme Pagnino le disait, ces affixes personnels sont des pronoms comme les autres. Oui, mais ils ne sont pas faits comme les autres.

L'exercice «d'inhonorificabilité» que propose Reuchlin est soigneusement choisi : le mot se découpe bien, sans trop tricher, et on croirait du Rabelais ${ }^{42}$. Il n'est pas surprenant que le concept d'affixe inventé par Reuchlin pour un objet morphologique spécifique (des marques personnelles suffixées) ait ensuite été généralisé : à mesure qu'on s'intéressait à des langues plus diverses, le vocabulaire trop dépendant de la morphologie du latin ou du grec allait parfois être senti comme

42 Rabelais évoque, Pantagruel VII (éd. 1542), la querelle de Reuchlin contre les dominicains de Cologne qui souhaitaient brûler les livres des juifs. Voir Price 2011. Dans la liste des livres que Pantagruel lit à la bibliothèque de Saint-Victor, on trouve les Tarraballationes doctorum Coloniensium adversus Reuchlin. On y trouve des mots comme Antipericatametanaparbeugedamphicribrationes. 
insuffisant, et souvent relégué à la sémantique, tandis que le vocabulaire expérimental créé lors des efforts de description de langues étrangères est mis à profit.

Mieux encore, comme le dira plus tard Schleicher, les langues 'agglutinantes' se révèlent être très communes, beaucoup plus que les langues flexionnelles ou isolantes, de sorte que le scalpel inventé par Reuchlin, l'outil de sa méthode analytique, se révèle aussi performant que la lunette de Galilée. Dès que les intellectuels des grandes puissances souhaiteront avoir des méthodes pour se donner la meilleure idée possible de la diversité des langues, au début du XIX ${ }^{\mathrm{e}}$ siècle, ils exploiteront à grande échelle les termes de 'racine', 'suffixe', 'préfixe', sur les pierres de dissection de l'anatomie des langues.

\section{OUVRAGES CITÉS}

Les éditions accessibles sur Google Books ou sur BNF-Gallica sont notées [GB] et [Gall].

\section{BIBLIOGRAPHIE}

\section{A. Éditions anciennes}

Buxtorf, Johannes, 1620. Epitome grammaticae hebraeae, breviter et methodice ad publicum scholarum usum proposita, Basileae, Editio 4a. (préface signée 1605).

- 1651. Thesaurus grammaticus linguae sanctae hebreae. Editio quinta, cum capitum et vocum irregularium indice, Basileae, inmpensis Heared, Ludovici regis, MDCLI. [GB]

Clenardus, Nicolas, 1534. Luah ha-diqduq. Tabula in grammaticen hebraeam, autore Nicolao Clenardo, Parisiis, Wechel. [GB, qui a aussi les éd. 1540, 1550, 1564]

Huet, Pierre Daniel, 1720. Dissertations sur différents sujets, composées par M. Huet. Recueillies par M. l'abbé de Tilladet, Tome premier, La Haye, Rogissart. [GB]

Isaacus (Levita), Iohannis, 1564. Grammatica hebraea, absolutissima, in duos libros distincta, Editio 4a, Antverpiae, Plantin. [GB]

Munsterus, Sebastianus, 1523. 'Aruk ha-šorašot. Dictionarium hebraicum, Autore F. Sebastiano Munstero minorita. Apud Frob. MDXXIII Mense Iunio. $(525$ p. +2$)$ [Gall]

- 1542. Mele'eket diqduq ha-šallem. Opus grammaticum consummatum ex variis Elianis [Elias Levitā] libris concinnatum, Autore Sebastiano Munstero [...], Basileae. [Gall, GB éd. 1549]

Münster 1548. Voir Qimhi David 1548.

Pagnino, Sante, 1549. Hebraicarum Institutionum libre IIII, Sancte Pagnino Lucensi authore, Ex R. Dauid Kimhi priore parte miklol, quam heleq ha-diqduq inscripsit, fere transcripti. [Gall éd. 1526, GB]

Pellikan (Pellican), Conrad, 1504. De Modo legendi et intelligendi hebraeum, Argentorati (Strasbourg) [Gall]

Petri Lombardi Parisiensis quondam episcopi Sententiarum magistri in totum Psalterium Commentarii, Parisiis anno 1541 data apud Poncetum Lepreux, sub Lupo, typis Joannis Lodoici Tiletani, in-folio minori cum privilegio regis et senatus. [GB]

Qimhî, David, 1540. Sep̄er Miklol ha-diqduq lešon ha-qodeš $r$. david qimhi ašer b-o otah kullah melammed u-mešallem, 'al yadei agatyu ha-qore' be-mișvat ha-melek elahit hamiqra’ bišibat pariṣ. Liber michlol Grammatices linguae sanctae R. Dauid Kimhi, quo eam integram docet et obsoluit, per Agathium Guidacerium, Parisiis, in Collegio Italorum, Anno 1540. (NB : ne contient que la $1^{\text {re }}$ partie : Morphologie du verbe) [GB] 
- 1545. Grammatica linguae hebraicae, auctore David Kimhi cum notis Eliae Levitae, Venetiis, Bomberg. In- ${ }^{\circ}, 269 \mathrm{f}$.

- 1548. Sēper ha-šsorašim 'im nigzarim. Dictionarium hebraicum iam ultimo ab autore Sebastiano Munstero recognitum, et ex Rabinis, praesertim ex Radicibus Dauid Kimhi, auctum et locupletatum, Basileae, Froben. Plus loin le titre : Dictionarium hebraicum, complectens radices cum derivatis. [BG, BNF a éd. 1539]

Qimhị, Mošeh, 1519. Mahalak (en hébreu ponctué). Rabi Mose Kimhi in introductorio grammaticae, Hagenau (À la fin, p. 78, il est nommé :) Sē̄er darkei lešon ha-qodeš le-r. mošeh qimhi (L'ouvrage est daté à la fin sur la page de la gravure : janvier 1519 et țevet 5279). Commence par : Mahalak ševilei ha-da'at... [GB]

- [+ Levita, trad. Munster], 1531. (texte en hébreu et en latin) Sep̄er diqduq hibro r. mošeh qimhi, 'im peiruš ha-rabbi eliyahu ha-levi aškenazi, Bâle, adar 5291 [1531] (Texte de la grammaire de Qimhi, ponctué : p. 1-51. L'autre partie du livre est la trad. lat. avec (en italique) la trad. lat. du commentaire de Levita.) $\{$ XIX-151\} (Cette éd. du texte hb, à 19 lignes/page, est différente de l'éd. en hébreu de 1520 au caractère plus rudimentaire, à 16 lignes/ page) [GB]

Reuchlin, Johannes, 1506. [Pas de page de titre] Ioannis Reuchlin Phorcensis LL.Doc. ad Dionysium fratrem suum germanum De Rudimentis Hebraicis. Liber primus. [Gall]

\section{B. Autres textes}

Allgeier, Arthur, 1951. «Ricardus Cenomanus und die Vulgata auf dem Konzil von Trient », Georg Schreiber (ed.), Das Weltkonzil von Trient, Freiburg, Herder, 359-380.

Apollonios Dyscole, 1997. De la Construction (syntaxe), Introduction, texte et traduction par Jean Lallot, Paris, Vrin, 2 vols.

Auroux, Sylvain (dir.), 1992. Histoire des idées linguistiques, vol. 2, Mardaga.

Bacher, Wilhelm, 1882. Die grammatische Terminologie des Jehuda B. Dawid Hajjûg, Wien, Carl Gerold's Sohn.

Bacher, Wilhelm, 1905. Die Bibel und Traditionexegetische Terminologie der Amoräer, Leipzig, Hinrichs.

Burnett, Stephen G., 1996. From Christian Hebraism to Jewish Studies. Johannes Buxtorf (1564-1629) and Hebrew Learnning in the Seventeenth century, Leiden, Brill.

Höpfel, Hildebrand, 1908. Kardinal Wilhelm Sirlets Annotationen zum Neuen Testament. Eine Verteidigung der Vulgata gegen Valla und Erasmus, Biblische Studien XIII/2. Freiburg im Breisgau, Herder.

Kessler-Mesguich, Sophie, 2013. Les Études hébraïques en France, de François Tissard à Richard Simon, Droz.

Price, David H., 2011. Johannes Reuchlin and the Campaign to Destroy Jewish Books, Oxford University Press.

Prijs, Leo, 1950. Die grammatikalische Terminologie des Abraham ibn Esra, Basel, SepherVerlag. 